\title{
Educación para el desarrollo sustentable en Chile: Deconstrucción pedagógica para una ciudadanía activa
}

Education for Sustainable Development in Chile: A Pedagogical

Deconstruction for an Active Citizenship

\author{
Volumen 20, Número 2 \\ Mayo - Agosto \\ pp. 1-26
}

Adolfo Berríos Villarroel

Jaime González Gamboa

\section{Citar este documento según modelo APA}

Berríos Villarroel, Adolfo. y González Gamboa, Jaime. (2020). Educación para el desarrollo sustentable en Chile: Deconstrucción pedagógica para una ciudadanía activa. Revista Actualidades Investigativas en Educación, 20(2), 1-26. Doi. 10.15517/aie.v20i2.41664 


\title{
Educación para el desarrollo sustentable en Chile: Deconstrucción pedagógica para una ciudadanía activa \\ Education for Sustainable Development in Chile: A Pedagogical Deconstruction for an Active Citizenship
}

\author{
Adolfo Berríos Villarroel ${ }^{1}$ \\ Jaime González Gamboa²
}

\begin{abstract}
Resumen: El presente ensayo analiza el panorama de la Educación para el desarrollo sustentable en Chile, para eso, examinamos diferentes propuestas de los organismos internacionales y, a su vez, cómo han sido adaptadas e implementadas en el sistema educacional chileno. El texto pone en tensión las bases epistemológicas que sustentan estas políticas "eco-educativas", invitando a deconstruir los cimientos del modelo de desarrollo sustentable y sus límites para el ejercicio de una ciudadanía activa en el contexto nacional. Para llevar a cabo esta reflexión el ensayo desarrolla, en un primer momento, los principales hitos del desarrollo sustentable en materia educativa, confrontando, en la discusión el concepto de ciudadanía que este programa propone, con el concepto desplegado en hitos relevantes del pensamiento político. Posteriormente, el análisis hace una propuesta sobre las herramientas que entrega la pedagogía crítica para develar la complejidad que implica abordar este modelo de desarrollo en la sala de clases. Para ello, nos basamos en los argumentos expuestos y en la pedagogía crítica de Paulo Freire. La reflexión final propone una perspectiva pedagógica que permita transformar el carácter instrumental que otorgan, actualmente, las actividades educativas a los futuros ciudadanos, con objeto de desarrollar una ciudadanía activa desde una perspectiva colectiva y local. Por último, el texto invita a cuestionar en qué medida la formación inicial de docentes del país contribuye a desarrollar las competencias pedagógicas necesarias para interrogar y enriquecer las recomendaciones ciudadanas propuestas en los programas educativos relacionados con el desarrollo sustentable.
\end{abstract}

Palabras clave: ciudadanía, desarrollo sustentable, pedagogía.

\begin{abstract}
The present essay analyses the outlook of the education for sustainable development in Chile. For that aim, we examine different proposals from international organizations, and, in turn, how these proposals have been adapted and introduced into the Chilean educational system. The epistemological fundaments that support these "eco-educational" politics are put in tension on this text, inviting to deconstruct the foundation of the sustainable development model, and its limitations to the practice of an active citizenship in the local context. In order to accomplish this reflection, the article firstly expounds on the primary landmarks of sustainable development in the educational subject, facing the concept of citizenship that this programme proposes, with the concept of citizenship displayed in the main landmarks of political thinking. Secondly, the analysis delivers a proposal regarding the tools given by critical pedagogy, in order to expose the complexity involved when dealing with the sustainable development model in the classroom. For this purpose, we base upon Paulo Freire's critical pedagogy's approach. As a final reflection, a pedagogical perspective is proposed, which allows a transformation of the instrumental nature given to future citizens by current educational activities, with the aim of developing an active citizenship from a collective and local perspective. Finally, the text prompts the question about the extent in which the early training of future Chilean teachers contributes to develop the necessary pedagogical competences, in order to examine and enrich the civic recommendations proposed in the educational programmes regarding sustainable development.
\end{abstract}

Keywords: citizenship, sustainable development, pedagogy.

\footnotetext{
${ }_{1}^{1}$ Académico de la Universidad Autónoma de Chile, en la Facultad de Educación, Chile. Doctor en Educación por la Université d'Aix-Marseille. Dirección electrónica: adolfo.berrios@uautonoma.cl Orcid https://orcid.org/0000-0002-2145-8067

${ }^{2}$ Académico adjunto de la Universidad de Talca y la Universidad Autónoma de Chile. Doctor en Filosofía por la Universidad de Chile.

Dirección electrónica: jaimgonzalez@utalca.cl Orcid : https://orcid.org/0000-0001-5385-3590
}

Ensayo recibido: 30 de octubre, 2019

Enviado a corrección: 14 de febrero, 2020

Aprobado: 27 de abril, 2020

Los contenidos de este artículo están bajo una licencia Creative Commons 


\section{Introducción}

Desde principios de la década de los años noventa, el sistema educacional chileno promueve el desarrollo sustentable ${ }^{3}$ como alternativa al actual modelo de desarrollo. Definido oficialmente por la Organización de Naciones Unidas (ONU) como un modelo de desarrollo capaz de satisfacer las necesidades del presente sin comprometer a las generaciones futuras de satisfacer las suyas (ONU, 1987), este plan propone, a causa de la escasez y agotamiento de los recursos naturales, encontrar un equilibrio entre el crecimiento económico, la satisfacción de las necesidades sociales y la preservación del medioambiente.

No obstante, si bien este plan de desarrollo se presenta como un compromiso internacional y consensuado frente a la crisis medioambiental, la puesta en práctica de sus objetivos provoca controversias entre diferentes protagonistas de la sociedad civil (políticos, empresariales, asociaciones ambientalistas, etc.). Una parte de la bibliografía denuncia el carácter híbrido de este término que intenta compatibilizar los imperativos de la economía y la preservación del medioambiente (Asara, Otero, Demaria, Corbera, 2015; Rist, 2012). Es decir, adoptando el paradigma dominante basado en la acumulación capitalista y el neoliberalismo, en términos económicos el desarrollo continúa midiéndose a través del Producto Interno Bruto (PIB). Por este motivo, según Theys (2014), el desarrollo sustentable puede considerarse como una manera de continuar con las políticas de crecimiento económico que, manteniendo el paradigma extractivista sobre la naturaleza, apela al progreso tecnocientífico como una forma de minimizar el impacto del sistema productivo en los ecosistemas terrestres.

Entonces, si el desarrollo sustentable considera el medioambiente como un reservorio de recursos naturales al servicio del sistema de producción industrial, surgen dudas hasta qué punto este proyecto político y económico constituye una finalidad educativa para abordar el actual escenario socioambiental del país (Sauvé y Orellana, 2014). Al respecto, cabe señalar que, en Chile, como en otros países del cono sur, la explotación de recursos minerales (cobre, litio, etc.) tiene un peso preponderante en la economía. Sin embargo, las consecuencias de las faenas mineras provocan diversos conflictos medioambientales que ponen en evidencia el débil control estatal en materia fiscal y en la gestión de desechos.

\footnotetext{
3 El término sustainable development ha sido traducido al español como desarrollo sostenible o desarrollo sustentable. Si bien algunos estudios argumentan diferencias entre los adjetivos "sustentable", "sostenible" y "sustentabilidad" (Gómez, 2014; Fernández y Gutiérrez, 2013). En el presente ensayo estos tres términos adosados al sustantivo desarrollo se emplean como sinónimos. Es decir, un modelo de desarrollo que busca generar un equilibrio entre los factores sociales, económicos y medioambientales para mejorar la calidad de vida de las personas.
} 
Además, según Gómez, Jiménez y Morán (2015), este panorama nacional se agudiza a raíz de los intereses económicos subyacentes al sistema democrático chileno, los cuales obstaculizan una redistribución justa de las utilidades económicas generadas por la explotación de estos recursos en el país.

Tomando en cuenta estos antecedentes, el presente ensayo busca problematizar y deconstruir el concepto de ciudadanía que el sistema escolar debería poner en marcha para cumplir con los objetivos de este modelo de desarrollo. De esta manera, la pregunta central es la siguiente: ¿en qué medida las actividades educativas contribuyen a formar una ciudadanía autónoma capaz de comprender, decidir y actuar frente a las necesidades que implican compatibilizar los imperativos del crecimiento económico y la reducción de la pobreza con la urgencia ecológica del cambio climático?

A partir de esta interrogante, este texto tiene como objeto analizar las políticas educativas chilenas relacionadas con la educación para la sustentabilidad, cuestionando las acciones que promueve el sistema escolar, para así generar propuestas pedagógicas que permitan empoderar a la comunidad escolar ante los desafíos que implica aportar a la constitución de una sociedad sustentable con ciudadanos realmente activos. Desde el ángulo de una educación para el desarrollo sustentable crítica y ciudadana (Barthes, 2017; Jickling y Wals, 2012), nuestro ensayo despliega un análisis que, más allá de adoptar comportamientos considerados a priori respetuosos del medioambiente por la ideología del desarrollo sustentable, invita a reflexionar sobre el enfoque pedagógico que sería necesario implementar para transformar el proyecto educativo de las Naciones Unidas en un verdadero proceso formativo, capaz de despertar el espíritu crítico de las personas que ejercerán la ciudadanía en la búsqueda autónoma de soluciones. Por lo tanto, este ensayo aporta elementos reflexivos para deconstruir el enfoque de ciudadanía promovido por la educación para el desarrollo sustentable, cuestionando en qué medida contribuye a una ciudadanía activa que adopte una posición política sobre los desafíos que enfrenta el país en esta materia.

\section{La educación al servicio del desarrollo sustentable}

Durante estos treinta años de trayectoria, diversas iniciativas han permitido sensibilizar a quienes son protagonistas de la educación con la fórmula de este plan de desarrollo.

La Cumbre de la Tierra, organizada en Río de Janeiro en 1992, contempla, en la Agenda XXI, que la institución escolar es fundamental para ejecutar el programa del 
desarrollo sustentable. A propósito, el capítulo 36 de este plan de acción señala que desarrollo y medioambiente son dilemas cruciales para las actuales y futuras generaciones. Por lo tanto, las intervenciones educativas no solo deben sensibilizar a la población sobre la protección del medioambiente, sino que también generar un cambio en sus comportamientos. A partir de entonces, las actividades pedagógicas relacionadas con la educación ambiental son progresivamente reorientadas en función del proyecto político de sustentabilidad propuesto por las Naciones Unidas (González, 2012).

En diciembre de 2002, la Asamblea General de las Naciones Unidas aprobó la resolución 57/254 estableciendo para los años 2005-2014 el Decenio de las Naciones Unidas de la Educación para el Desarrollo Sostenible. A cargo de la UNESCO, este decenio invita a los estados a poner en práctica estrategias y planes de acción nacional para alcanzar los objetivos del desarrollo sustentable. El objetivo principal es incorporar en el proceso de enseñanza-aprendizaje los temas claves de la sustentabilidad, tales como: recursos naturales, derechos humanos, cambio climático, biodiversidad, pobreza, salud, etc. A nivel pedagógico, se enfatiza la necesidad de incorporar este programa en todas las asignaturas y ciclos del sistema educativo. Además, destaca la necesidad de implementar un enfoque interdisciplinario y sistémico que permita comprender la interdependencia de factores sociales, económicos y medioambientales que intervienen en este proyecto de desarrollo (Unesco, 2009).

La Agenda 2030 para el Desarrollo Sustentable es la nueva estrategia internacional para hacer realidad la propuesta de Naciones Unidas. Para los próximos quince años, este plan propone como objetivos principales: la reducción de la pobreza y la desigualdad social dentro y entre los países, la construcción de sociedades pacíficas e inclusivas, la protección de los derechos humanos, la igualdad de género y la protección del planeta y sus recursos, entre otros (ONU, 2015). En este ambicioso plan, la educación se transforma en el motor que puede acelerar o retrasar el paso hacia una ciudadanía con modos de vida sostenibles. Al respecto, el cuarto objetivo de la Agenda 2030 enfatiza la necesidad de una educación inclusiva y equitativa para crear calidad. Por eso, el sistema educativo debe proporcionar acceso a los conocimientos teóricos y generar experiencias de aprendizaje para promover las ideas del desarrollo sustentable entre las nuevas generaciones. 


\subsection{Panorama de la educación para la sustentabilidad en el sistema escolar chileno}

$\mathrm{Si}$ bien estos tres programas internacionales contribuyen progresivamente a la elaboración de la educación para la sustentabilidad, este proyecto educativo se construyó sobre una serie de actividades y políticas públicas propias de cada país. En este sentido, Girault, Zwang y Jeziorski (2013) señalan que los textos de Naciones Unidas y la Unesco se acomodan al contexto político, económico y cultural.

En el caso de Chile, la retórica que siguió a la Conferencia de Río en 1992 se impone paulatinamente en un sistema escolar heredero de las políticas de descentralización y privatización implementadas en los años ochenta (Cox, 2011). El debilitamiento institucional que estas reformas provocan en el Ministerio de Educación (MINEDUC) —en el contexto del decenio de una Educación para el Desarrollo Sustentable (2004-2015) - implica que hasta el día de hoy se ejecutan diversos programas ministeriales relacionados con la sustentabilidad. En este marco de iniciativas se encuentra el programa País de eficiencia energética iniciado en el año 2005 por el Ministerio de Economía, y continuado cinco años más tarde por el Ministerio de la Energía. Posteriormente, con la creación del Ministerio del Medioambiente (MMA) en el año 2010, la división de educación ambiental y participación ciudadana llevan a cabo actividades para introducir los objetivos de la sustentabilidad en la sala de clases. En esta línea, destaca el Sistema Nacional de Certificación Ambiental de Establecimientos Educacionales (SNCAE), que utiliza el discurso de la sustentabilidad para promover, tanto a nivel pedagógico como en la gestión administrativa, el desarrollo de hábitos y conductas de vida que permitan mejorar la protección del medioambiente.

Para coordinar este mosaico de experiencias educativas relacionadas con la sustentabilidad, la Política Nacional para el Desarrollo Sustentable (2009) es la primera estrategia nacional que entrega directrices metodológicas para formar una "ciudadanía" que participe activamente en el cumplimiento de los objetivos del desarrollo sustentable en el país. Portando la etiqueta del MMA, este plan propone: el pensamiento sistémico e interdisciplinario, la capacidad de diálogo y mediación para resolver problemas, el trabajo en equipo, la capacidad de toma de decisiones, entre otros. Desde un punto de vista didáctico, estas metodologías orientan los procesos educativos para "formar personas reflexivas, responsables éticamente y con capacidad de actuar en el presente, pensando en el futuro y en el bien común" (Ministerio de Medio Ambiente, 2009, p. 13). 
Ratificando su compromiso con el plan de desarrollo de Naciones Unidas, el nuevo marco legal de la educación chilena, la ley n²0.370 (2009), Ley General de Educación (LGE), incorpora la sustentabilidad como uno de los principios fundamentales que inspiran la educación nacional. Al respecto, este principio exhorta al sistema educativo a llevar a cabo actividades que favorezcan el respeto del medioambiente y el uso racional de los recursos naturales, considerados como una forma de solidaridad con las futuras generaciones.

Si bien, la reforma curricular de mediados de los años noventa introduce el principio de desarrollo sustentable entre los Objetivos Fundamentales Transversales de los diferentes ciclos educativos, hay que esperar hasta el año 2013 para que el Mineduc elabore un documento institucional propio acerca de la visión educativa nacional sobre la sustentabilidad. Considerando el propósito de abordar transversalmente el desarrollo sustentable, se incita a la comunidad educativa a trabajar los objetivos de este modelo de desarrollo en todas las asignaturas y quehaceres de la escuela. El propósito fundamental es fortalecer la formación ciudadana mediante conocimientos, actitudes y habilidades para todas las personas de la comunidad educativa. Al respecto, el Mineduc plantea:

(...) tomar decisiones conscientes y responsables frente a las problemáticas sociales, ambientales, culturales y económicas, y que éstas tienen consecuencias, positivas o negativas, que los implican, así como a la familia, al barrio, la escuela o el jardín infantil, la comuna o el país. (Mineduc, 2013, p. 5)

Aunque las nuevas bases curriculares de educación parvularia (2018), básica (2018a) y media (2015) intentan aminorar el retraso que han experimentado las políticas educativas implementadas por el Mineduc en materia de sustentabilidad, surgen interrogantes sobre el desarrollo de capacidades que favorezcan el pensamiento autónomo y crítico en esta materia. Si bien, no existen estudios científicos que permitan evaluar el alcance de los contenidos relacionados con el desarrollo sustentable incluidos en el nuevo curriculum escolar, el acomodo del programa de la Unesco en el sistema educativo nacional entrega algunos elementos de análisis que es necesario revisar por su contribución a la formación ciudadana del estudiantado.

Al respecto, la revisión bibliográfica realizada por Salinas (2016) evidencia que, a pesar de que existen programas para abordar las problemáticas ambientales en las aulas de la educación formal chilena como el SNCAE, su implementación depende de las voluntades del 
profesorado y el personal directivo, por lo tanto, queda en duda su aporte real para el análisis del contexto natural y social del país.

Para Berríos y Martínez (2016), el rol que ocupan los programas de los ministerios de Energía y Medioambiente revelan una apropiación parcial y reducida de las problemáticas que implica el desarrollo sustentable. En efecto, las prácticas educativas de estos programas apuntan principalmente al desarrollo de comportamientos favorables al medioambiente por medio de la utilización responsable de la energía y la gestión de los desechos. En este sentido, los ecogestos, como economizar el consumo de energía eléctrica o el reciclaje, por útiles que sean como herramientas de sensibilización, no permiten comprender de manera global el desarrollo sustentable con sus dimensiones sociales, económicas y políticas. Además, si consideramos la lógica neoliberal de este modelo de desarrollo, las herramientas que entrega el sistema educativo nacional no favorecen la formación de una ciudadanía capaz de reflexionar sobre los desafíos que implica la puesta en práctica de este proyecto. En este sentido, sin la entrega de saberes científicos que permitan apoyar la reflexión y la capacidad argumentativa de agentes escolares, surgen dudas respecto de si la sala de clases se transforma realmente en un espacio de discusión para participar en las decisiones públicas que implica construir una sociedad sustentable.

Entonces, considerando que en los programas educativos chilenos los contenidos relacionados con el desarrollo sustentable se focalizan más bien en la entrega de prácticas sociales de comportamiento, las clases pueden adquirir una tendencia normativa donde la frontera entre manipulación ideológica y educación es frágil. Es decir, las acciones y comportamientos a ejecutar en el marco de la sustentabilidad suponen una reflexión previa profunda, por ende, necesitan, más allá de su aspecto motivacional, un componente cognitivo y ético. Por lo tanto, es conveniente deconstruir la dimensión ciudadana propuesta por este concepto para generar una propuesta pedagógica que permita tomar distancia sobre la visión del mundo que conlleva este modelo de desarrollo, basada en la acumulación de riquezas y el crecimiento económico.

\section{Sobre los orígenes de la ciudadanía}

Aristóteles en La Política sostiene que la ciudadanía es la puesta en práctica de la libertad -eleuthería- (Aristóteles, trad. en 1986, pp. 107-127) que, es la capacidad de generar un proyecto y, por consecuencia, supone las posibilidades para tomar decisiones de una 
persona, una comunidad u organización en relación con su entorno cultural, social y medioambiental.

La estrecha relación entre ciudadanía y libertad, implica la forja del carácter (ethos) (Cortina, 2013) que, permite desplegar una comprensión de los acontecimientos relevantes para el individuo y la ciudad, al tenor de las virtudes que conducen el juicio y la decisión hacia la búsqueda del mejoramiento de la convivencia. En este sentido, la virtud -areté(Aristóteles, trad. en 2001, pp. 75-93) se entiende como la capacidad reflexiva y práctica de templar el carácter para deliberar con otros respecto a la vida buena para la pólis. Por lo tanto, la virtud ciudadana determina la forma del ser humano desde su participación en el mundo común sin que este pierda su singularidad.

La concepción de Aristóteles tiene continuidad en el pensamiento moderno de Kant (2002), pues este mantiene la idea de la ciudadanía como una capacidad humana que se constituye en la praxis, es decir, es en el ejercicio público de la razón (Kant, 1994), en donde el sujeto, a partir de la reflexión y la ilustración, es capaz de dar contenido a su voluntad para ejercer su autonomía.

La ciudadanía no es una característica aledaña al sujeto, es, más bien, fundamental, puesto que permite crear el interés ${ }^{4}$, un espacio en el que convergen diferentes modos de ser que resguardan al ser humano en su pluralidad dentro de la ciudad y más allá de ella. Es un concepto que sintoniza y también rebasa el límite de los derechos establecidos por la producción de la ley, puesto que está en relación con la ley basada en la moralidad -la ley moral- (Kant, 1993). Esta supone que todo ser racional mundano e, incluso, de latitudes desconocidas es capaz de evaluar una situación por sí mismo y puede darse las leyes a sí mismo. Esto es, puede juzgar un hecho injusto para luego llevar a cabo una acción de intervención de esa situación; ejemplo de eso es la crítica que Kant hace en el texto El Conflicto de las Facultades. En este desarrolla la idea de que la facultad de filosofía no debía recibir preceptos de ninguna otra facultad o gobernante temporal, comprendiendo que esta debe sostener la autonomía de poder reflexionar, pensar y examinar, más allá de los poderes particulares que la circundan. Ello le dará imparcialidad y serenidad para observar y comprender los fenómenos del mundo y del acto de consciencia que le permitirá conocer y actuar.

Desde estas miradas, el concepto de "ciudadanía" que se articula a partir de los convenios internacionales y en la recepción de estos con las políticas educativas en vista del

${ }^{4}$ Palabra que viene del latín inter-esse, que remite a un ser que se abre desde la pluralidad. 
desarrollo sustentable, instalan una ciudadanía que contradice el mismo concepto, pues encauza preceptos y prácticas que mantienen un velo de irreflexión y, por consiguiente, promueven una visión que podría ir en contra de los intereses del territorio y sus comunidades locales. Por ello, al comprender la actividad ciudadana desde esa matriz, se ponen en marcha lo que comprendemos como una ciudadanía inocua que se mueve en un campo de posibilidades predecibles que no van al fondo del problema. Esta manera de comprender la actividad ciudadana neutraliza su potencial crítico y contiene un componente cínico, entendiendo este término como la performance ciudadana de anular su propio principio y potencial transformador. Por ende, se abre una brecha entre individuos "globalizadores" y "globalizados" (Dobson, 2005, p. 54), pues los primeros son agentes de acción y, los segundos, en cierta medida, receptores pasivos que no hacen el ejercicio real de ser ciudadanos y ciudadanas.

En seguida, retomaremos las ideas claves del concepto de ciudadanía desplegado en momentos claves del pensamiento político para deconstruir la propuesta ciudadana del desarrollo sustentable:

A. La contradicción principal que tiene el concepto de ciudadanía medioambiental prescrito en el informe Brundtland de 1987 y en todas las políticas que desde él se desprenden, contiene en su núcleo, la neutralización de una posible ciudadanía activa, pues condiciona la autonomía de los ciudadanos y de las ciudadanas, subordinándolo a sostener esa praxis en un marco incuestionable, obliterando la posibilidad de repensar las bases sobre las que el modelo se instala.

B. El modelo neoliberal sobre el que se instala el desarrollo sustentable se caracteriza por el diagnóstico de algunos autores ordoliberales que, para explicar el caudillismo y los diferentes totalitarismos de la segunda guerra mundial, pusieron como causa de ese problema al Estado (Bilger, 1965; Foucault, 2004). Eso supuso pensar y crear un orden en que la administración del gobierno y de la libertad no estuviera en manos del Estado, sino en una estructura mayor que superara los "vicios" de este. Es decir, se invierte la tesis anterior, para instaurar un orden en que la libertad del mercado es la que debe primar sobre el Estado. Desplazamiento clave, que puede ser comprendido como la economización del mundo o como la era de la razón economicista, pues agudiza un tipo de poder cada vez menos centrado en instituciones clásicas y busca materializar a un ser humano basado en el individualismo extremo que, para 
desarrollar su libertad, debe exacerbar la competitividad consigo mismo y con el otro para lograr satisfacer sus necesidades.

C. Entonces, el velo de irreflexión que tendría la ciudadanía medioambiental en el marco del desarrollo sustentable, es que no profundiza con suficiente fuerza el modelo donde se gesta, sino que se orienta a generar acciones individuales como una forma de convivir harmónicamente con los ecosistemas terrestres. Ahora, si bien el informe Brundtland expone una crítica al crecimiento económico, a nuestro juicio, este adolece de superficialidad, pues no cuestiona el andamiaje neoliberal sobre el que ese crecimiento económico se erige. El crecimiento económico pasó de ser un medio a ser un fin en sí mismo, un nudo irreflexivo y doctrinario (Rodríguez, Sanhueza, 2014). Por lo tanto, la tierra es considerada como un medio o un instrumento a través del cual se pueden obtener recursos y acumular riquezas.

D. En este sentido, para que la ciudadanía medioambiental no sea innocua y concrete sus posibilidades reales, es necesario, en nuestra perspectiva, abrir el campo educativo para que la praxis ciudadana inicie su proceso desde la capacidad reflexiva en que cada persona es capaz de dar contenido a su voluntad para integrarse con otros y generar las transformaciones necesarias para la protección del ecosistema. Eso significa ir más allá de los gestos ecopolíticos rebosantes de sonrisas, para construir, desde las necesidades propias de las comunidades locales (cosmovisiones, valores, niveles de educación, etc.), las políticas medioambientales que estén en una relación de equilibrio con el entorno. Un ejemplo icónico, entre muchos otros casos, del desajuste entre modelo de desarrollo y medio ambiente es la fractura que provoca la desmesura de intereses comerciales en Chile que, amparados en un neoliberalismo dogmático, impiden la satisfacción de la necesidad básica del acceso al agua, modificando completamente el panorama socio-ambiental de la provincia de Petorca (Bolados, 2018).

E. Citemos el informe Brundtland:

En términos generales, es evidente que, si no se satisfacen las necesidades básicas de los seres humanos, la preocupación por el medio ambiente es secundaria. El ser humano tiene que sobrevivir, responder y preocuparse en primer lugar de sus necesidades básicas -alimentos, vivienda, condiciones sanitarias- y, después, del medio ambiente. (ONU, 1987, p. 280) 
La cita está en tensión con el enunciado del informe que define el desarrollo sustentable como un equilibrio entre el crecimiento económico, la satisfacción de las necesidades sociales y la preservación del medioambiente. Ello, porque en los países en vías de desarrollo, la aplicación del marco neoliberal en que el informe se inserta implica un desequilibrio entre lo público y lo privado, entre los bienes de uso común y las propiedades particulares que generan desigualdad social. Esta, a su vez, dificulta el progreso económico, fragiliza la democracia, obstaculiza la convivencia y amenaza la cohesión social (PNUD, 2017).

Así, tenemos un binomio clave para comprender la estructura del desarrollo sustentable que, de no ser repensado, seguirá siendo obstáculo para una posible ciudadanía medioambiental. Esta es, la necesidad de cuestionar el modelo como un factor que determina la profundización de la desigualdad y, esta última, como una barrera fundamental para aplicar, en las políticas educativas, la ciudadanía activa. Es decir, el ejercicio soberano de la libertad que potencia la interdependencia entre lo económico, las necesidades sociales y el cuidado del medio ambiente se fisura, pues se desequilibra esta triada, en favor del crecimiento económico desregulado e irracional.

\subsection{Hacia una ciudadanía activa}

El concepto de ciudadanía que subyace a las políticas emanadas de los acuerdos internacionales para el desarrollo sustentable, despliega una particular forma de entender esta praxis; se enfoca en un tipo de ciudadanía pasiva que establece concesiones respecto al desarrollo basado en un modelo utilitarista.

Con esto último, nos referimos a dos elementos claves, por una parte, está el principio de racionalidad utilitaria que reza que el individuo solo busca la utilidad egoísta y, por otra parte, que solo busca obtener el máximo beneficio social. Estos componentes son parte de la concepción de un individuo que en sus acciones se esmera por conseguir logros hedonistas, desvinculados de la idea de bien común, si no es para usarla como medio o instrumento para conseguir un objetivo, bien o servicio. Esta visión se condensa en la idea de Bentham (1789), quien dice que una política pública se justifica en cuanto significa llevar a cabo el mayor beneficio posible a la mayor cantidad de personas.

Ante este planteamiento, la óptica de Sen (2000) dice que el principio de racionalidad utilitarista es insuficiente para comprender las motivaciones de las personas, pues hay múltiples acciones en que estas actúan con un sentido de bien común, como por ejemplo, el 
voto, las diferentes acciones solidarias, etc., en que la personas que los ejercen buscan colaborar mancomunadamente, trascendiendo la idea de una inframotivación egoísta.

Junto a esto, Sen (2010), en su propuesta, se enfoca en la crítica a la idea de crecimiento económico, un modelo estrictamente técnico y desvinculado de la ética (Conill, 2017), en que el nivel de comprensión respecto a la sociedad, familia e individuo se evalúa desde el producto interno bruto. Para este autor, se trata más bien, de abrir múltiples dimensiones que permitan observar cómo los bienes y servicios afectan a las personas, es decir, cómo estos permiten a las personas crear y desarrollar capacidades para el uso de las oportunidades que contribuyan al proyecto de vida. Por lo tanto, se supera la idea de crear productos que tienen solo por finalidad ser consumidos, sino que, se enfatiza el proceso a través del cual ese producto genera un impacto en el individuo, sociedad, medio ambiente y organización.

En este sentido, para Sen, entre los factores que permiten el desarrollo de la libertad, junto con la educación y la salud, está la capacidad ciudadana, entendida desde la información y la libertad de expresión que, deben sostenerse en la discusión para profundizar la comprensión de los temas que afectan la vida. Así, la persona puede tomar las mejores decisiones y participar activamente en la superación de los problemas de su entorno socio-cultural y medio-ambiental.

Ahora bien, si pasamos por el cedazo del enfoque sobre la libertad de Sen, esta idea del desarrollo sustentable, atisbamos que requiere de un examen crítico en que se devele su carácter a priori respecto a su aplicación en los países en vías de desarrollo, lo que abre inquietudes como ¿cuál es el nivel de participación de las comunidades locales para el diseño de este modelo? ¿qué clase de libertad es la que se expresa en las personas que están subordinados a este paradigma? ¿cuál es el sentido de la educación para construir — desde este modelo- las sociedades futuras?

A partir de estas preguntas, la que se orienta hacia la educación es crucial, puesto que es la posibilidad más relevante para poner en marcha los valores que puedan forjar el carácter (Cortina, 2011). No obstante, nos encontramos con el problema que se asienta en el objetivo que el desarrollo sustentable da a esta: generar un sistema de reproducción del modelo desarrollista utilitario, promoviendo una particular forma de comprender el bienestar humano desde la competitividad exacerbada respecto a lograr objetivos que eleven el estatuto del individuo en la sociedad. Bienestar que, hasta el momento, se construye en la 
idea de producir bienes, servicios y cosas, con objeto de multiplicar las necesidades, medios y fines para satisfacerlas "infinitamente".

En este sentido, Esposito (2002) nos dice que el instante en que la "libertad" estalla con mayor vigor, es decir, cuando estamos en el exceso de libertad de un mercado que, a través de la competencia, reduce a la libre elección del consumidor las posibilidades subjetivas de la libertad, es el momento en que la libertad se restringe inéditamente en la historia humana.

El ser humano está fijado a estructuras de poder donde el totalitarismo de la libertad técnico-capitalista nos interpela a repensar la relación con la política y con la educación, en la medida que dicha expresión de libertad -como elección bursátil, consumista y empresarialinocula la libertad pensada desde la ciudadanía activa.

Con este panorama, si el desarrollo sustentable busca generar una consciencia real respecto al cuidado y conservación del medio ambiente ¿cómo puede llevar a cabo dicha pretensión si no es cuestionando el sistema de necesidades que elabora productos como si los recursos fueran infinitos? ¿de qué forma es posible construir una ciudadanía activa cuando esta se encuentra en un estado de minoría de edad pues las condiciones dadas para su ejercicio sustentable neutralizan sus capacidades reales?

\section{Enfoque crítico y ciudadano de la sustentabilidad: tensiones y desafíos}

Manteniendo la postura crítica frente a las ambigüedades que envuelven este concepto, diversos estudios realizados en el área francófona invitan a las personas que protagonizan el mundo de la educación a tomar distancia, e insisten en la necesidad de implementar metodologías educativas que examinen las controversias que este modelo de desarrollo genera a nivel local e internacional (Barthes, Lange y Tutiaux-Guillon, 2017; Hertig, 2016; Lange, 2014).

En esta línea de trabajo, Legardez y Simonneaux (2011) califican las temáticas del desarrollo sustentable (energías renovables, bio-combustibles, igualdad social, etc.) como problemáticas socialmente relevantes. Para estos autores, son temas controversiales que, teniendo en cuenta la heterogeneidad de las fuentes de referencia, generan discusiones entre la comunidad de científicos. Además, suelen ser mediatizados por la prensa y, por lo tanto, pueden influenciar los saberes previos de las personas que participan en la comunidad escolar. Considerando sus alcances sociales, enfrentar estos temas en la sala de clases implica complementar los saberes disciplinarios con los valores e ideologías que acompañan 
las decisiones relacionadas con la sustentabilidad. De esta manera, comprender las problemáticas del desarrollo sustentable, por una parte, exige la implementación de estrategias de aprendizaje activas (debates, método de proyecto, etc.) que permitan al estudiantado reflexionar sobre los dilemas que plantea este modelo de desarrollo. Desde este punto de vista, la educación para la sustentabilidad se inscribe bajo una perspectiva crítica y ciudadana que invita, mediante la integración de las controversias y pluralidad de interpretaciones, a construir argumentos propios como ciudadanos y ciudadanas de una sociedad democrática.

Por otra parte, Jickling y Wals (2008) señalan que la educación para la sustentabilidad puede oscilar entre un enfoque transmisivo y transformador. En el primer caso, la educación sirve como puente para transmitir de manera unilateral las ideas definidas por los grupos de poder (políticos, empresarios) en relación con el desarrollo sustentable. En este sentido, este modelo es propuesto como la única vía correcta a seguir y toda acción sobre el medioambiente se ajusta a la lógica político-económica de este modelo de desarrollo. En el segundo caso, en el enfoque transformador, el saber es co-construido por las personas que movilizan la comunidad escolar, como consecuencia, está influenciado por sus saberes previos y referentes culturales. En este enfoque, el objetivo es generar una ciudadanía crítica capaz de cuestionar el mundo actual y de participar. De esta forma, el propósito principal es formar ciudadanos y ciudadanas capaces de reflexionar con otros y otras para elaborar decisiones comunes, allí donde la sustentabilidad se presenta como una alternativa más.

Si bien el enfoque transformador y socio-constructivista parece ser el más adecuado para abordar la naturaleza compleja y controversial del desarrollo sustentable, elaborar estrategias metodológicas de este tipo no es algo evidente en la sala de clases, y representa un desafío didáctico para las prácticas habituales del profesorado. Cabe señalar al respecto, que el término desarrollo sustentable no es un concepto científico que puede ser anexado a una disciplina en particular, más bien, se trata de un compromiso político-económico entre los estados que permite conservar el lazo implícito entre las políticas neoliberales y la idea de progreso, por lo que su legitimidad a nivel de las referencias científicas es heterogénea (Barthes, Alpe y Bader (2013). Entonces, puesto que en el proceso de transposición didáctica que acompaña los contenidos relacionados con la sustentabilidad, los saberes sabios no son la única fuente de referencia para transformarlos en objeto de la enseñanza ¿cuáles son los saberes que la persona docente puede utilizar como referencia? 
Aunque las personas docentes pueden apoyarse en otro tipo de saberes como son los saberes institucionales, estudios sobre el curriculum escolar de la educación para el desarrollo sustentable en Latinoamérica (Flores, García y Rayas, 2017) y en España (Sureda, Catalán, Álvarez y Comas 2013), indican que la perspectiva extractivista y antropocéntrica del medioambiente está presente en los programas oficiales, promoviendo una lectura consensual de este modelo de desarrollo. Por consiguiente, la escolarización de los contenidos relacionados con el desarrollo sustentable no contribuye a comprender la complejidad del plan de las Naciones Unidas para el siglo XXI, puesto que las contradicciones y controversias que implica su puesta en práctica no aparecen como una herramienta que puede enriquecer la formación ciudadana del estudiantado.

De esta manera, si consideramos que el discurso del desarrollo sustentable refleja una sola manera de interpretar la interrelación sociedad, economía y medioambiente, la postura neutra e imparcial que debe guiar el quehacer docente en el contexto de la educación escolar chilena esconde un trasfondo político. De hecho, sin saberlo, las actividades relacionadas con la educación para la sustentabilidad se transforman en un acto político y una toma de posición que transmiten una sola manera de interpretar el mundo. Por consecuencia, el enfoque transformador y socioconstructivista puede generar tensión en el trabajo que lleva a cabo el cuerpo docente. Es decir, llevar a la sala de clase las discusiones que rodean los proyectos relacionados con la sustentabilidad en el país (extracciones mineras, forestales, pesca artesanal, etc.), los valores y convicciones personales docentes que pueden afectar las actividades educativas. Por consiguiente ¿cómo enseñar una temática que es objeto de debate y controversia?

\section{Pistas pedagógicas para transformar la educación para la sustentabilidad en vector de cambio social}

Al retomar los fundamentos relacionados con la ideología político-económica dominante que considera la noción desarrollo sustentable, la educación no puede limitarse solo a su labor de difusión y sensibilización mediante la promoción de gestos y comportamientos relacionados con la protección del medioambiente. Por el contrario, es necesario dotar el acto educativo de una pedagogía crítica que entregue, a docentes y a estudiantes, herramientas epistemológicas, cognitivas y actitudinales para cuestionar y tomar decisiones acerca de los alcances del modelo de desarrollo propuesto por la ONU. 
Según Bazán (2002), la pedagogía crítica considera la escuela no solo como un espacio de socialización heredera de la sociedad, sino que también como un lugar que permite repensar y reflexionar sobre el funcionamiento del sistema democrático para así construir una sociedad mejor. En esta pedagogía, la reflexión, la emancipación y la transformación constituyen tres principios claves y pueden motivar la implementación de metodologías que contribuyan a cuestionar la viabilidad a nivel local del plan de sustentabilidad propuesta por las Naciones Unidas. En este sentido, Cayul y Bazán (2014) proponen que la pedagogía crítica del sur entrega a las personas docentes herramientas que les permiten incorporar el contexto local en sus prácticas educativas, como una plataforma para cuestionar el discurso hegemónico y los intereses económicos, políticos y culturales de la clase dominante. Es decir, esta perspectiva pedagógica permite incorporar la diversidad presente en el contexto, así como los diferentes puntos de vistas de los distintos actores sociales.

De este modo, el debate, la indagación y el pensamiento crítico (Gómez, Royo, 2015; Olivo, 2017) sobre las controversias que genera la implementación de este proyecto políticoeconómico en el país, son recursos que podrían contribuir a desenmascarar el discurso oficial. En palabras de Paulo Freire (2009), estas herramientas permitirían transformar el carácter instrumental que ocupan los actores escolares chilenos en este proyecto educativo. Asimismo, considerando que la búsqueda de soluciones no está reservada solo a los expertos y que, por consiguiente, no existe una única solución válida y racional, la educación para la sustentabilidad se transforma en una educación para la libertad que involucra al profesorado y al estudiantado en su calidad de ciudadanos y ciudadanas.

Desde este punto de vista, en un primer instante, es clave re-politizar el término desarrollo sustentable y liberar el concepto de libertad ciudadana puesto que es una praxis pedagógica ineludible en la sala de clases. Aludiendo a su raíz griega, politikós, la palabra política hace referencia a quienes ejercen la ciudadanía. Es decir, se entiende que los objetivos propuestos por este modelo de desarrollo exigen previamente la formación de capacidades ciudadanas que favorezcan sus capacidades argumentativas y de compromiso, de manera que les permita participar en los debates públicos y en las transformaciones que persigue la agenda de sustentabilidad de las Naciones Unidas.

Desarrollar estas capacidades implica, en un primer instante, cuestionar los discursos internacionales y nacionales en favor de este modelo de desarrollo como una respuesta natural a la crisis socio-ecológica que afecta el planeta. De esta forma, se pone en marcha 
una pedagogía crítica que invita a los actores escolares a dudar de las proposiciones que emergen del sector político y económico, así como de sus alianzas y la utilización del relato científico para justificar la movilización ciudadana en favor de la protección del medioambiente. Para Bader (2011), se trata de promover, a través de este ejercicio pedagógico, una educación para la sustentabilidad crítica que favorezca la reflexión a propósito de las ideologías que configuran nuestra manera de concebir el mundo y el medioambiente, para así fomentar un cambio social. Así, interrogantes tales como: ¿quién decide y en nombre de qué? o ¿para qué? deben preceder a toda práctica educativa considerada como sustentable.

En un segundo instante, y una vez instalada la duda, la re-politización incentiva a reflexionar en qué medida el desarrollo sustentable es una verdadera alternativa al modelo capitalista y sus abusos. En el contexto económico actual de mundialización y sobreexplotación de los recursos naturales, vale la pena examinar si este plan de desarrollo es una transformación profunda del actual sistema o, más bien, solo una readecuación cosmética que maquilla el modelo. Al respecto, podríamos señalar que el carácter extractivo del desarrollo sustentable que se apoya en la explotación de los recursos naturales como base del sistema productivo, homogeniza la manera de interpretar la relación ser humanonaturaleza propagado por la ideología económica dominante. Esta uniformización invisibiliza las diferencias y no otorga espacios para abordar la relación sociedad-naturaleza-economía desde otro ángulo, como es el caso del Buen Vivir (Avendaño, 2010), de la libertad como desarrollo de capacidades (Sen, 2000) y del vínculo entre economía y ética (Conill, 2017). De esta forma, prevalece una sola ideología que plantea un modelo de desarrollo que a nivel discursivo se considera alternativo, puesto que, intenta atenuar los efectos medioambientales y sociales que provoca el crecimiento económico. Sin embargo, en la realidad cotidiana este discurso se diluye y marginaliza las estrategias de resistencia que emergen desde la sociedad civil como la economía solidaria, los huertos colectivos, etc. (González, 2017).

Siguiendo a Sousa Santos (2010), la epistemología del sur puede contribuir a generar una ecología de saberes que integre no solo los conocimientos y las formas de comprender el mundo impuestos por la cultura eurocéntrica-occidental, sino que también los saberes que han sido marginalizados y oprimidos por la dinámica neo-colonialista de esta cultura. Desde este punto de vista, esta epistemología es una herramienta descolonizadora que puede contribuir a evidenciar el sesgo racionalista-técnico y economicista que, utiliza el término 
desarrollo sustentable para interpretar la relación del ser humano con los ecosistemas terrestres y los seres vivos que los habitan. Considerando los diferentes proyectos, que desde 1992 a la fecha, han colaborado progresivamente a propagar los objetivos de la sustentabilidad (Agenda XXI, Decenio de la educación para el desarrollo sustentable, Agenda 2030 para el desarrollo sustentable), la propuesta de Sousa Santos es una alternativa epistemológica que nutre las prácticas pedagógicas con herramientas teóricas que inviten a reflexionar sobre el grado de libertad que otorga la dimensión educativa del modelo de desarrollo defendido por las Naciones Unidas

Estos dos pasos previos son esenciales para llevar a cabo una pedagogía crítica que contribuya a despejar la niebla ideológica que cubre al discurso tecno-economicista del desarrollo sustentable. Aunque la innovación tecnológica en materia de nuevas fuentes de energía es fundamental para disminuir el impacto del crecimiento económico en el medioambiente, resulta necesario también educar a las personas sobre las causas que motivan la búsqueda de nuevas fuentes de energía, ello supone repensar la actividad ciudadana desde el potencial emancipatorio que esta tiene: la actividad de repensar el territorio, las fuentes de energía y, junto a esas, la jerarquización de necesidades para vivir en armonía con el medio ambiente. Es decir, sin un proceso educativo que invite a reflexionar sobre el impacto de nuestro modo de vida en el medioambiente, la apología por el desarrollo de nuevas tecnologías puede transformarse en una invitación para continuar con nuestras conductas cotidianas. Es más, se perpetúa la creencia de que los recursos del planeta son ilimitados y se olvida que las nuevas tecnologías, supuestamente amigables con el medioambiente, son más bien una respuesta provisoria para afrontar la crisis socioecológica que experimenta el planeta.

Este pensamiento técnico que prevalece en la propuesta del desarrollo sustentable para pensar los cambios y adaptaciones que implica el antropoceno, tiende a desestimar la responsabilidad individual y colectiva en un modelo que promueve, explícita e implícitamente el sobreconsumo y, por ende, exige un flujo de energía exacerbada. En efecto, sin una pedagogía ciudadana capaz de favorecer una vigilancia crítica del efecto que tiene este credo en nuestras conductas, se vuelve difícil salir del binomio consumir-desechar.

Considerando que la educación para la sustentabilidad exhorta a docentes y estudiantes a adherir a un discurso preestablecido y adoptar compartimentos favorables a la instauración de este modelo de desarrollo, más que nunca es necesario rescatar las ideas de Freire (2008) sobre el poder de la ideología en la enseñanza. Según el autor brasileño, la 
práctica educativa docente exige estar alerta al encubrimiento de los hechos y a la miopía de que ciertas ideas pueden afectar el quehacer pedagógico. En este sentido, toda actividad pedagógica, en el marco de este proyecto educativo, requiere una actitud de desconfianza y crítica de parte de la persona docente, "quien no se siente dueño de la verdad ni tampoco objeto adaptado al discurso ajeno que le es dictado autoritariamente" (Freire, 2008, p.128).

Por lo tanto, antes de llevar a la práctica nuevas propuestas tecnológicas favorables a los objetivos del desarrollo sustentable, es pertinente que el propósito pedagógico instale dispositivos de duda en torno a la premisa de que el avance científico y tecnológico favorece el bienestar de los seres humanos y la convivencia de estos con el resto de los seres vivos, y no el afán de lucro de la minoría que dirige el planeta. En otras palabras, la alocución al progreso tecnológico como pedal para avanzar en la construcción de una sociedad sustentable tiene implicancias éticas y políticas que no se pueden eludir y que es necesario abordarłas en la sala de clases.

Entonces, la transición hacia un modelo ecológicamente compatible con la preservación del medioambiente es inviable si la responsabilidad de los mecanismos que regulan el funcionamiento del sistema económico está ausente de la discusión ético-política. Por ejemplo, cuestionar en qué medida los intereses de las multinacionales son compatibles con los objetivos del desarrollo sustentable; si sus actividades pueden considerarse sustentables tomando en cuenta su aspiración a la maximización en la obtención de beneficios en el mediano y corto plazo. Esto significa implementar una pedagogía que permita contraponer sus intereses con la escasez de los recursos naturales y a la vez visualizar sus repercusiones sociales. Por ende, abordar los objetivos del desarrollo sustentable en la sala de clases obliga a problematizar de manera conjunta las tensiones que provocan la economía y la política sobre el medioambiente. Este ejercicio reflexivo es fundamental para analizar la desconfianza hacia las instituciones democráticas tradicionales, las cuales se muestran ineficaces para abordar las problemáticas que genera el actual modelo de desarrollo.

Como consecuencia, esta perspectiva pedagógica crítica y reflexiva que abre espacios a las controversias y discusiones, constituye una herramienta para perforar el ciego dogmatismo que rodea al discurso del desarrollo sustentable. En este sentido, la invitación a debatir los alcances y dilemas de los objetivos de este modelo de desarrollo se transforma en un ejercicio democrático que contribuye a explicitar sus paradojas. Precisamente, es a partir de estas fisuras que aparecen entre el discurso y la práctica, donde están las claves para 
despertar y educar el espíritu crítico del estudiantado sobre este modelo de desarrollo. Se trata, entonces, de generar un espacio de discusión abierto, es decir, libre de la alienación del desarrollo sustentable, para cuestionar las posibilidades que tiene la participación ciudadana frente al "imperativo" del crecimiento económico.

\section{Reflexiones finales}

Para concluir, es necesario poner en escena los principales argumentos, que van en el sentido de deconstruir los cimientos sobre los que el modelo de desarrollo sustentable se instala para abrir las tensiones entre una ciudadanía subordinada y una ciudadanía medioambiental crítica, que cimiente el camino para la formación de docentes y de una ciudadanía activa.

Para ello, retomaremos el binomio clave para comprender la estructura del desarrollo sustentable, que es el obstáculo para una posible ciudadanía medioambiental. A saber, la necesidad de preguntarnos y cuestionar el modelo como un factor que determina la profundización de la desigualdad y, ésta como una barrera fundamental para aplicar, en las políticas educativas, la ciudadanía activa. El ejercicio soberano de la libertad que potencia la interdependencia de la triada compuesta por crecimiento económico, la satisfacción de las necesidades sociales y el cuidado del medio ambiente se quiebra, pues se desequilibra en favor del crecimiento económico dogmático y desregulado.

Eso supone realizar una crítica al modelo neoliberal sobre el que se instala el desarrollo sustentable, pues este implica un desplazamiento crucial que puede ser comprendido como la economización del mundo, pues profundiza un tipo de poder cada vez menos centrado en instituciones clásicas y busca materializar a un ser humano basado en el individualismo extremo que, para desarrollar "su libertad", debe exacerbar la competitividad y la relación con un sistema de consumo en permanente expansión.

De este modo, si la triada del desarrollo sustentable se fractura, por lo tanto, el concepto de ciudadanía allí involucrado, nace sin el carácter necesario para responder a sus concepciones y prácticas efectivas. Con esto, nos referimos a que en sus orígenes la ciudadanía medioambiental contiene una libertad subordinada a patrones de desarrollo que no responden a los intereses del territorio y de las comunidades locales, puesto que estos, más bien sirven como instrumentos para la acumulación de riquezas y para reproducir la desigualdad en una relación patológica con el medio natural. 
Por consecuencia, la educación ciudadana para la sustentabilidad restringe la capacidad de decisión y de acción de las personas que protagonizan el sistema escolar chileno a un margen estrecho. Su función educativa y formativa se limita, más bien, a prepararlas para tomar decisiones con anticipación, como si se tratara de responder a un cuestionario con preguntas cerradas, entendiendo que esta forma de comprender el ejercicio de la ciudadanía está circunscrita a la supuesta convivencia harmónica de la triada antes mencionada, sin posibilidades de cuestionar el marco sobre el que esta se funda. En nuestra óptica, esto es una contradicción vital, pues no mira el problema de frente y nutre, con abonos poco confiables, la puesta en práctica de una "libertad" condicionada desde sus bases.

Sobre este condicionamiento, el "diálogo" se abre a veces para debatir cuestiones de orden técnico y no sobre los fundamentos del proyecto de sociedad que este modelo pretende construir en el país. Este tipo de educación está condenada a tener un carácter instrumental, centrada en la enseñanza de ecogestos (reciclaje, uso responsable del agua potable, energía eléctrica, etc.) que, si bien tienen un impacto para sensibilizar a las nuevas generaciones, son insuficientes para hacer frente a los desafíos sociales y medioambientales que están en juego. Además, arraiga la responsabilidad en la construcción de una sociedad sustentable a nivel individual y no colectivo, que por tanto, tiende a promover el hedonismo individual, articulado sobre la base del hiperconsumismo que rebasa las necesidades reales.

Considerando la ausencia de debates sobre las repercusiones del programa de desarrollo sustentable de las Naciones Unidas, la pedagogía crítica se revela como una herramienta capaz de develar el trasfondo político que encierra esta noción. Esta pedagogía reorientaría el proceso educativo ligado a la sustentabilidad, disminuyendo su actual carácter normativo y técnico para favorecer el desarrollo de una crítica social que permita reflexionar sobre el verdadero lugar que ocupa la ciudadanía en el diseño de una sociedad sustentable. Con este enfoque, la educación para el desarrollo sustentable adquiere una dimensión crítica y enriquece la formación ciudadana, puesto que considera las reflexiones y necesidades locales de la comunidad educativa cuestionando las relaciones de poder verticalistas, a través de la confianza y del respeto por los saberes de cada una de las subjetividades que configuran el proceso de enseñanza-aprendizaje. Es decir, más que un fin en sí, el término desarrollo sustentable se transforma en un medio dinámico para cuestionar las posibilidades que ofrece la educación formal, para pensar y deconstruir una sociedad más justa, equitativa y solidaria. 
El desarrollo sustentable es una idea todavía abierta, en que el profesorado, a través de su praxis pedagógica, pueden problematizar y transformar los nudos irreflexivos que este contiene. Ello tensiona la idea de una pedagogía basada en la posición dogmática respecto de una verdad incuestionable y "objetiva”, para desplegar la construcción social de la verdad, en que el profesorado discuta, proyecte, muestre su sensibilidad, sus valores y convicciones personales ante los problemas de sustentabilidad.

En consecuencia, es bajo este enfoque que la educación para la sustentabilidad puede adquirir un carácter emancipador y ciudadano que contribuya a cuestionar la realidad social y medioambiental que el modelo de desarrollo instaura. Así, es fundamental incorporar esta visión pedagógica en la formación inicial docente, pues son precisamente las generaciones actuales y aquellas del mañana, quienes experimentarán las trasformaciones que persiguen los objetivos del desarrollo sustentable. Sin la preparación adecuada del cuerpo docente para llevar a cabo estrategias pedagógicas de este tipo, las actividades educativas, en el contexto de la educación para el desarrollo sustentable, continuarán siendo aisladas y neutralizadas respecto a la reflexión de fondo que se evidencia en la siguiente pregunta: ¿En qué medida la educación permite el desarrollo de capacidades ciudadanas que permitan transformar a las nuevas generaciones en personas ecociudadanas activas de nuestro planeta?

\section{Referencias}

Aristóteles. (1986). Política. Madrid, Alianza Editorial.

Aristóteles. (2001). Ética a Nicómaco. Madrid, Alianza Editorial.

Asara, Viviana., Otero, Lago., Demaria, Federico. y Corbera, Esteve. (2015). Socially sustainable degrowth as a social-ecological transformation: repoliticizing sustainability. Sustainability Science, 10(3), 375-384. Doi: https://doi.org/10.1007/s11625-015-0321-9.

Avendaño, Octavio. (2010). El buen vivir. Una vía para el desarrollo. Revista Polis Latinoamericana, 9(25), 557-561.

Bader, Bárbara. (2011). Education à l"environnement dans une société du risque : la conception des sciences privilégiée de l"éducation au développement durable. En B. Bader \& L. Sauvé (Eds.), Education, environnement et développement durable : vers une écocitoyenneté critique (pp. 223-250). Québec, Canadá: Presses de l'université Laval.

Barthes, Angela. (2017). Quels outils curriculaires pour des « éducations à » vers une citoyenneté politique ? Educations, International sciences and technical edition, ISTE, 17(1), 25-40. Recuperado de https://www.openscience.fr/Numero-1-327 
Barthes, Angela., Alpe, Yves. y Bader, Bárbara. (2013). Questions and positions on education for sustainable development at university in France: Example of short professional cycles. Environmental Education Research, 19(3), 269-281.

Barthes, Angela., Lange, Jean-Marc. y Tutiaux-Guillon, Nicole. (2017). Dictionnaire critique des enjeux et concepts des «éducations à». Paris, Francia: l'Harmattan.

Bazán, Domingo. (2002). Pedagogía social y pedagogía crítica: nexos y fundamentos básicos. Paulo Freire. Revista de Pedagogía crítica, 1, 49-61. Recuperado de http://bibliotecadigital.academia.cl/bitstream/handle/123456789/1609/049061.pdf?sequence $=1$ \&isAllowed $=y$

Bentham, Jeremy. (1789). Los Principios de la Moral y la Legislación. Madrid: Edición Francisco Ferrer y Valls.

Berrios, Adolfo. y Martínez, Enrique. (2016). Quelques pistes pour gagner le pari de l'Éducation au développement durable au Chili. Education Relative à l'Environnement Regards, Recherches, Réflexions, 13(2). Recuperado de https://journals.openedition.org/ere/801

Bilger, François. (1965). La pensé économique libérale dans l'Allemagne contemporaine. [Texto expuesto en la 29a reunión de trabajo de estudios del centro de estudios políticos y cívicos. (C.E.P.E.C.)]. Paris.

Bolados, Paola. (2018). La naturaleza política de la sequía en Petorca. Ciper. Recuperado de https://ciperchile.cl/2018/04/27/la-naturaleza-politica-de-la-sequia-en-petorca/

Cayul, Miguel. y Bazán, Domingo. (2014). Hacia una pedagogía crítica del sur: caminos, atajos y desvíos necesarios de recorrer. Paulo Freire. Revista de Pedagogía crítica, 15, 103-118. Recuperado de http://revistas.academia.cl/index.php/pfr/article/view/384/492

Conill Sancho, Jesús. (2017). Nuevos horizontes de economía ética en tiempo de neurociencia. Pensamiento, 73(276), 541-562. Recuperado de https://proyectoscio.ucv.es/wp-content/uploads/2017/09/20-Nuevos-horizontes_JesúsConill-Sancho.pdf doi: pen.v73.i276.y2017.020

Cortina Orts, Adela. (2011). Neuroética y neuropolítica: sugerencias para la educación moral. Madrid: Editorial Tecnos.

Cortina Orts, Adela. (2013). ¿Para qué sirve la Ética?. Barcelona: Editorial Paidós.

Cox, Cristián. (2011). Currículo escolar de Chile: génesis, implementación y desarrollo. Revue International de Education de Sevres, 56, 1-9. Recuperado de: http://mapeal.cippec.org/wp-content/uploads/2014/06/Curr\%C3\%ADculo-escolar-deChile-g\%C3\%A9nesis-implementaci\%C3\%B3n-y-desarrollo.pdf

Dobson, Andrew. (2005). Ciudadanía Ecológica. Isegoría 32, 47-62. Recuperado de https://philpapers.org/rec/DOBCE-2 
Esposito, Roberto. (2002). Immunitas. Protezione e negazione della vita. Torino: Ed. Einaudi.

Fernández, Lilia y Gutiérrez, Mirella. (2013). Social, Economic and Environmental Welfare for Present and Future Generations. Información tecnológica, 24(2), 121-130. Recuperado de https://dx.doi.org/10.4067/S0718-07642013000200013

Flores, R., García, M. y Rayas, J. (2017). La educación ambiental en la formación docente inicial en México. Pesquisa em Educação Ambiental, 12(2), 80-92.

Foucault, Michel. (2004). Naissance de la Biopolitique. Paris: Ed. Gallimard.

Freire, Paulo. (2008). Pedagogía de la autonomía. México: Siglo XXI.

Freire, Paulo. (2009). Pedagogía del compromiso. América Latina y Educación Popular. Barcelona: Hipatia Editorial.

Girault, Yves., Zwang, Aurélie y Agnieska, Jeziorski. (2013). Finalités et valeurs de différentes politiques d'ESD. Education Relative à l'Environnement - Regards, Recherches, Réflexions, 11, 61-80.

Gómez, Jennifer. (2014). Del desarrollo sostenible a la sustentabilidad ambiental. Revista Facultad de Ciencias Económicas: Investigación y Reflexión, 22(1), 115-136.

Gómez, Juan., Jiménez, Juan y Morán Dalmiro. (2015). El impacto fiscal de la explotación de los recursos naturales no renovables en los países de América Latina y el Caribe. Santiago, Chile: Comisión Económica para América Latina y el Caribe (CEPAL).

Gómez, Verónica., Royo, Paulina. (2015). Construyendo espacios de diálogo: una propuesta de enseñanza de la bioética. Acta Bioethica, 21(1), 9-18.

González-Gaudiano, Édgar. (2012). La ambientalización del curriculum escolar: breve recuento de una azarosa historia. Profesorado. Revista de curriculum y Formación Profesorado, 16(2), 15-24.

González, Raúl. (2017). Ensayo sobre economía colaborativa, solidaria y autogestionaria. Hacia una economía plural. Santiago, Chile: Ediciones Forja.

Hertig, Philippe. (2016). Des outils de pensée pour appréhender la complexité dans le cadre de l'éducation en vue du développement durable. En M. Ethier y E. Mottet (Eds.), Didactiques de l'histoire, de la géographie et de l'éducation à la citoyenneté. Recherches et pratiques (pp. 117-128). Bruxelles: De Boeck.

Jickling, Bob. y Wals, Arjen. (2008). Globalization and environmental education: looking beyond sustainable development. Journal of Curriculum Studies, 40(1), 1-21.

Jickling, Bob. y Wals, Arjen. (2012). Debating Education for Sustainable Development 20 Years after Rio: A Conversation between Bob Jickling and Arjen Wals. Journal of Education for Sustainable Development, 6(1), 49-57. 
Kant, Immanuel. (1993). Crítica de la razón práctica. Buenos Aires: Editorial Losada.

Kant, Immanuel. (1994). Filosofía de la historia. México. D.F.: Editorial Fondo de cultura económica.

Kant, Immanuel. (2002). El conflicto de las facultades. (Colección pedagógica universitaria), 37-38. Recuperado de https://cdigital.uv.mx/handle/123456789/5690

Lange, Jean-Marc. (2014). Éducation au développement durable: intérêts et limites d'un usage scolaire des investigations multiréférentielles d'enjeux. Éducation et socialisation, 36 DOI : 10.4000/edso.959

Legardez, Alan y Simonneaux, Laurence. (2011). Développement durable et autres questions d'actualité. Questions socialement vives dans l'enseignement et la formation. Dijon: Educagri Editions.

Ministerio de Educación. (2013). Cómo llegamos a ser una comunidad educativa sustentable. Recuperado de http://sustentabilidad.umce.cl/wp-content/uploads/2016/10/MINEDUC2013-comunidad educativa sustentable.pdf

Ministerio de Educación. (2015). Bases curriculares $7^{\circ}$ básico a $2^{\circ}$ año medio. Recuperado de https://media.mineduc.cl/wp-content/uploads/sites/28/2017/07/Bases-Curriculares7\%C2\%BA-b\%C3\%A1sico-a-2\%C2\%BA-medio.pdf

Ministerio de Educación. (2018). Bases curriculares de Educación parvularia. Recuperado de https://parvularia.mineduc.cl/wpcontent/uploads/sites/34/2018/03/Bases Curriculares Ed Parvularia 2018.pdf

Ministerio de Educación. (2018a). Bases curriculares primero a sexto básico. Recuperado de https://www.curriculumnacional.cl/614/articles-22394 bases.pdf

Ministerio del Medio Ambiente. (2009). Política Nacional de Educación para el Desarrollo Sustentable. Recuperado de https://educacion.mma.gob.cl/wpcontent/uploads/2017/10/PNEDS-PDF.pdf

Olivo, María. (2017). Educación para la ciudadanía en Chile. Información Tecnológica, 28(5), 151-164.

Organización de las Naciones Unidas para la Educación, la Ciencia y la Cultura. (2009). United Nations Decade of Education for Sustainable Development (DESD, 2005-2014). Review of Contexts and Structures for Education for Sustainable Development. Recuperado de http://www.unesco.org/pv obj cache/pv obj id CE807503B33BC2DDA327F2368FAD 728369440B00/filename/justpublished desd2009.pdf.

Organización de Naciones Unidas. (1987). Informe de la Comisión Mundial sobre el Medio ambiente $y$ el desarrollo. Recuperado de: http://www.ecominga.uqam.ca/PDF/BIBLIOGRAPHIE/GUIDE LECTURE 1/CMMADInforme-Comision-Brundtland-sobre-Medio-Ambiente-Desarrollo.pdf 
Organización de Naciones Unidas. (2015). Transformar nuestro mundo: la Agenda 2030 para el Desarrollo Sostenible. Recuperado de http://www.un.org/es/comun/docs/?symbol=A/69/L.85

Rist, Gilbert. (2012). Le développement: histoire d'une croyance occidentale. (4⿳亠丷厂 ed.). Paris, Francia: Presses de Sciences Po.

Rodríguez, Ignacio y Sanhueza, Paulina. (2014). ¿Es racional fomentar el crecimiento económico?. Revista latinoamericana Polis, 13(37), 499-520.

Salinas, Denisse. (2016). Educación ambiental para el desarrollo y consumo sustentable en Chile. Una revisión bibliográfica. Revista electrónica Educare, 20(2), 1-15. Recuperado de https://www.revistas.una.ac.cr/index.php/EDUCARE/article/view/7647/16495

Sauvé, Lucie y Orellana, Isabel. (2014). Entre développement durable et vivir bien : repères pour un projet politico-pédagogique. Revue internationale d'éthique sociétale et gouvernementale. 16(1). Recuperado de https://journals.openedition.org/ethiquepublique/1406

Sen, Amartya (2000). Desarrollo y libertad. Barcelona Editorial Planeta.

Sousa Santos, B. (2010). Descolonizar el saber, reinventar el poder. Montevideo, Uruguay: Ediciones Trilce.

Sureda, Jaume., Catalán, Albert., Álvarez, Olaya y Comas, Rubén. (2013). El concepto de "desarrollo sostenible" en la regulación del currículo de la Educación Secundaria Obligatoria en España. Estudios pedagógicos (Valdivia), 39(1), 253-267. Doi : https://dx.doi.org/10.4067/S0718-07052013000100015

Theys, Jacques. (2014). Le développement durable face à sa crise: un concept menacé, sous-exploité ou dépassé? Développement durable \& territoires, 5(1). Recuperado de https://journals.openedition.org/developpementdurable/10196 
Revista indizada en

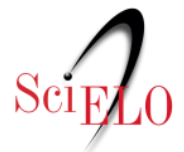

redalyc satindex

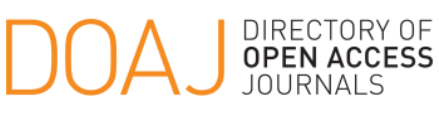

Distribuida en las bases de datos:

- Dialnet

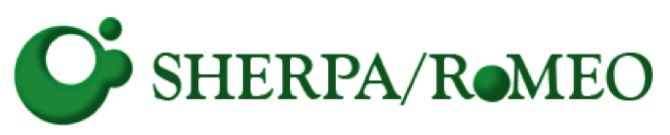

REDIB

Red Iberoamericana

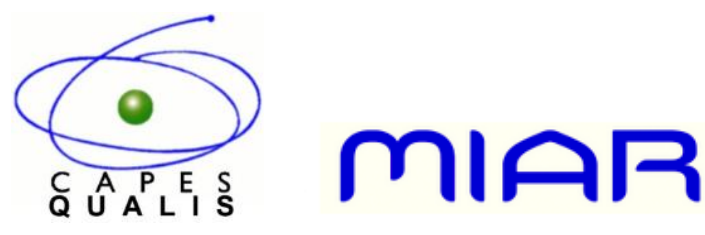

